\title{
A population-based study of inflammatory mechanisms and pain sensitivity
}

\author{
Elina lordanova Schistad ${ }^{\mathrm{a}, \star}$, Xiang Yi Kong $^{\mathrm{b}, \mathrm{c}}$, Anne-Sofie Furberg $^{\mathrm{d}, \mathrm{e}}$, Emmanuel Bä Ekryd $^{\mathrm{f}, \mathrm{g}}$, Guri Grimnes ${ }^{\mathrm{h}, \mathrm{i}}$, \\ Nina Emaus ${ }^{j}$, Leiv Arne Rosseland ${ }^{a, k}$, Torsten Gordh', Audun Stubhaug ${ }^{a, k}$, Bo Engdahlm ${ }^{m}$, Bente Halvorsen ${ }^{b, c}$, \\ Christopher Sivert Nielsen ${ }^{m}$
}

\begin{abstract}
Two recent studies suggest that experimental pain sensitivity is associated with low-grade systemic inflammation. However, only 2 biomarkers have been identified, and the studies were conducted in adult individuals where confounding effects of comorbid diseases cannot be excluded. We therefore tested associations between pain sensitivity and 119 inflammation-related serum biomarkers in 827 healthy adolescents (15-19 years) in the population-based Troms $\varnothing$ Study: Fit Futures. The main outcome measure was cold-pressor pain tolerance (CPT), tested by placing the dominant hand in circulating cold $\left(3^{\circ} \mathrm{C}\right)$ water for a maximum of 105 seconds. Secondary outcomes were heat and pressure pain threshold and tolerance. Twelve proteins and 6 fatty acids were significantly associated with CPT after adjustment for possible confounding factors and correction for multiple comparisons. Of these, all fatty acids and 10 proteins were protective, ie, higher biomarkers levels were associated with increased CPT, whereas 2 biomarkers were associated with lower tolerance. Taken together, these biomarkers predicted completion of the tolerance test with a C-statistic of 0.65 . Results for heat and pressure pain tolerance were remarkably similar, strengthening the generalizability of our findings. In this cohort of young healthy individuals, we found a relationship between inflammation-related biomarkers and pain tolerance and thresholds. Biomarkers with anti-inflammatory and analgesic effects predominated, suggesting that the development of prophylactic dietary or pharmaceutical treatments may be possible.
\end{abstract}

Keywords: Pain sensitivity, Cold-pressor, Inflammation, Biomarkers

\section{Introduction}

Systemic inflammation is a causative factorinchronic pain. This is most obviously true for autoimmune diseases, such as rheumatoid arthritis, where immune activity has a direct effect on tissue pathology..$^{19}$ However, there is increasing evidence that inflammatory mechanisms are implicated in chronic pain conditions outside the autoimmune domain. Notably, increased levels of

\footnotetext{
a Division of Critical Care, Oslo University Hospital Rikshospitalet and Ulleval, Oslo, Norway, ${ }^{b}$ Research Institute of Internal Medicine, Oslo University Hospital Rikshospitalet, Oslo, Norway, ' Institute of Clinical Medicine, Medical Faculty, University of Oslo, Oslo, Norway, ${ }^{\circ}$ Department of Community Medicine, Faculty of Health Sciences, UiT The Arctic University of Norway, Tromsø, Norway, ${ }^{e}$ Department of Microbiology and Infection Control, University Hospital of North Norway, Tromsø, Norway, ${ }^{f}$ Pain and Rehabilitation Centre, University Hospital, Linkoping, Sweden, ${ }^{g}$ Department of Medical and Health Sciences, Linkoping University, Linko ping, Sweden, " Tromsø Endocrine Research Group, Institute of Clinical Medicine, UiTThe Arctic University of Norway, Tromsø, Norway, 'Division of Internal Medicine, University Hospital of North Norway, Tromsø, Norway, ${ }^{j}$ Department of Health and Care Sciences, Faculty of Health Sciences, UiT The Arctic University of Norway, Tromsø, Norway, ${ }^{~}$ Pain Research Group, Institute of Clinical Medicine, Medical Faculty, University of Oslo, Oslo, Norway, ' Department of Surgical Sciences/Pain Research, Uppsala University, Uppsala, Sweden, ${ }^{m}$ Department of Chronic Disease and Ageing, Norwegian Institute of Public Health, Oslo, Norway

*Corresponding author. Address: Department of Physical Medicine and Rehabilitation, Oslo University Hospital HF, Ulleval, Postbox 4956 Nydalen, 0424 Oslo, Norway.Tel.:14723027441.E-mailaddress: uxioel@ous-hs.no(E.I.Schistad).
}

proinflammatory cytokines have been found in fibromyalgia, chronic whiplash-associated disorder, and irritable bowel syndrome, where objectively measurable tissue pathology is absent. $3,10,17,24,34$ However, most such studies are small, and the track record of replication is correspondingly poor. For instance, a systematic review found that although 2 or more studies reported elevated serum levels of interleukin (IL)-1RA, IL6 , and IL-8 in fibromyalgia, meta-analysis was significant for IL-6 only. ${ }^{36}$ In addition to sample size, many of these studies do not control for lifestyle factors. This is particularly problematic in studies of clinical pain, as chronic pain may lead to insomnia, deconditioning, and obesity, which contribute to altered immune activity. 2,29 There is therefore a real possibility of reverse causality. Clinical case studies arealso unable to distinguish effects that are specific to the disease itself, such as cartilage degeneration in osteoarthritis, from more general neuroplastic changes in the peripheral and central nervous system. This is of major importance, as twin studies have found that the genetic mechanisms are largely the same for different pain phenotypes, ${ }^{38,39}$ suggesting that common molecular mechanisms can be identified, and treatments that span traditional diagnostic boundaries can be developed.

Epidemiological studies of experimental pain sensitivity circumvent or reduce many of the above limitations. They are typically large, can adequately control for lifestyle factors, and the possibility of reverse causality is considerably reduced. Moreover, associations between experimental pain and biomarkers that are observed in healthy individuals are more likely to reflect neuroplastic changes than tissue-specific pathology. Finally, human experimental pain paradigms have close analogues in animal 
models, where there is extensive evidence that neuroinflammation induces neuroplastic changes resulting in central sensitization and widespread hyperalgesia. ${ }^{20,21,31,32}$ Descending pain modulatory systems arising in the brainstem are important in the maintenance of chronic pain and generalized hyperalgesia. Parallel findings in human studies of experimental pain would greatly increase the plausibility of generalizing from animals to humans. To the best of ourknowledge, only 2 large-scale studies have examined relationships between inflammation and human experimental pain sensitivity. One found a positive correlation between cold-pressor tolerance and $C$-reactive protein (CRP) in a large adult sample. ${ }^{30}$ A second study, found that the $v-3$ resolvin precursor 17-HDHA was associated with increased heat pain thresholds in a sample of adult twins. ${ }^{37}$ Although both studies suggest that pain sensitivity is associated with chronic low-grade inflammation, the number of biomarkers examined is limited. Furthermore, although the first of these studies controlled for chronic pain and lifestyle factors, residual confounding from acute pain conditions and nonpainful chronic conditions that affect pain sensitivity (eg, nonpainful neuropathies) cannot be excluded. Consequently, we aimed to analyse associations between a large number of proinflammatory and antiinflammatory biomarkers in a cohort of healthy adolescents.

\section{Methods}

\subsection{Study design and population: Fit Futures}

In 2010 to 2011, all first-year upper-secondary school students in 2 neighbouring municipalities in northern Norway attended the Tromsø Study: Fit Futures. ${ }^{40}$ The invited cohort included 1117 adolescents, of which 1038 (530 boys) participated in the survey, providing an attendance rate of $92.9 \%$ (Fig. 1). Participants aged - 19 years, and adolescents with cognitive disabilities were

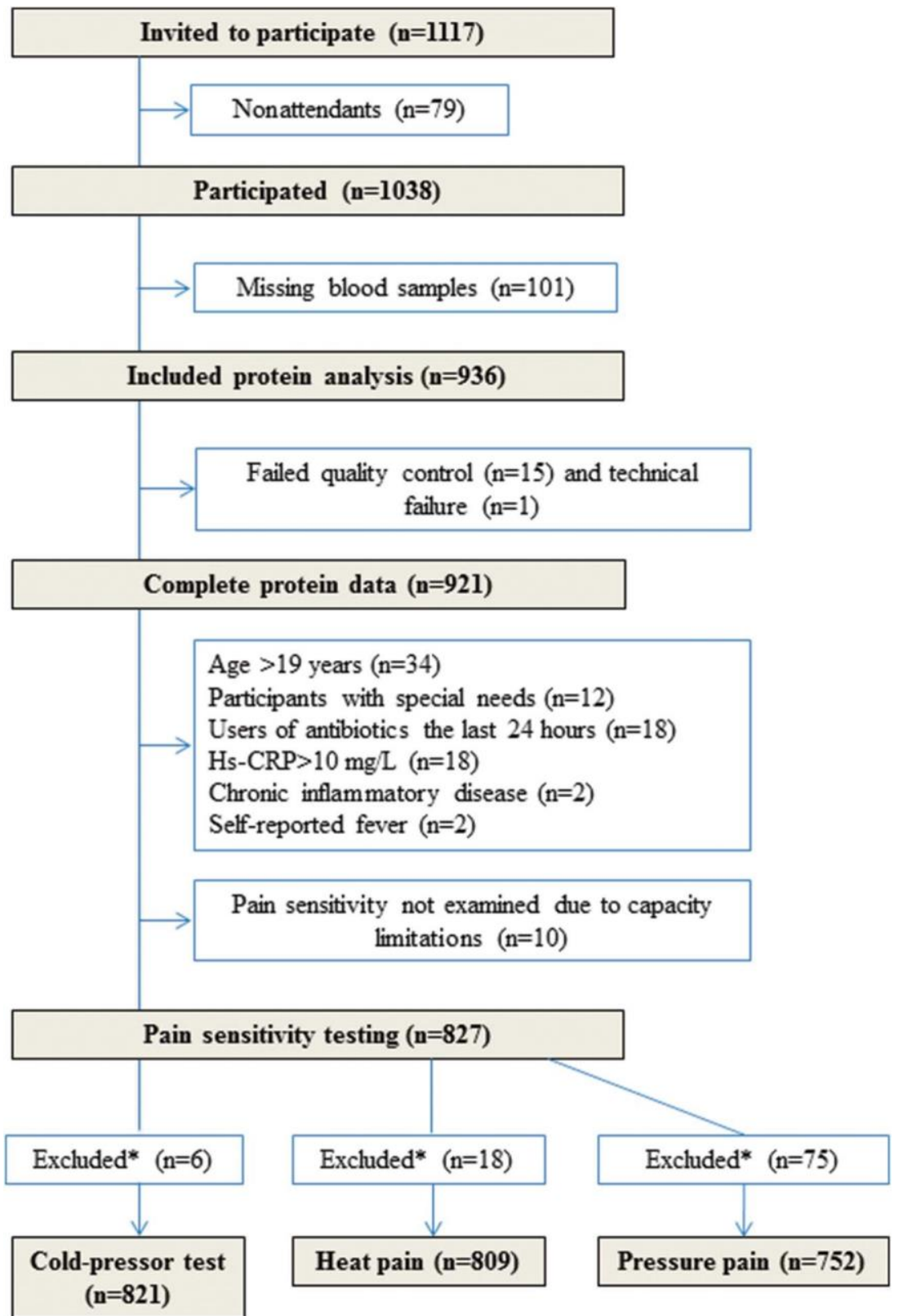

Figure 1. Study flowchart—-the Tromsø Study, Fit Futures. *Excluded due to technical/procedural failures, medical reasons or lack of comprehension. 
excluded from the analysis due to possibly diverging biology, behaviour, orunderstanding of the testinstructions. Furthermore, participants with inflammatory arthritis, fever, antibiotic use in the last 24 hours, or participants with high-sensitivity CRP - $10 \mathrm{mg} / \mathrm{L}$, which mirror otheracute orchronic diseases, were also excluded.

\subsection{Study assessments}

\subsubsection{Clinical interview and questionnaires}

Informationaboutmedical history, medication use, fever, orother symptoms of ongoing infection were obtained through a clinical interview performed by trained nurses at the Clinical Research Department, University Hospital of North Norway, Tromsø. All reported chronic diseases were encoded according to ICD-10 nomenclature. Height and weight were measured to the nearest $0.1 \mathrm{~cm}$ and $0.1 \mathrm{~kg}$, respectively, on an electronic scale (Jenix DS 102 stadiometer; Dong Sahn Jenix, Co, Ltd, Seoul, Korea) with the participants wearing light clothing and no shoes. Body mass index (BMI) was calculated using the formula weight/height ${ }^{2}$ $\left(\mathrm{kg} / \mathrm{m}^{2}\right)$. Participants filled in a web-based questionnaire on lifestyle and general health, including pubertal development. No girls were pregnant. Participants were classified regarding their tobacco use into 4 categories: Nonusers of any tobacco products; users of snuff only; smokers only; and users of both snuff and smoke. The short version of the Alcohol Use Disorders Identification Test for Consumption (AUDIT-C) was used for screening of alcohol misuse, and cutoff scores of 7 for boys and 5 for girls were used. ${ }^{11}$ The participants indicated their level of leisure-time physical activity in the pastyearusing 1 of 4 response categories: sedentary: reading, watching television, or engaging in sedentary activities; light: at least 4 hours a week walking, bicycling, or engaging in other types of physical activity; moderate: at least 4 hours a week exercising to keep fit and participating in recreational athletics; and vigorous: regular, vigorous training or participating in competitive sports several times a week. The Short Mood and Feelings Questionnaire (SMFQ) was used to screen for depressive symptoms, and a cutoff score of $\$ 11$ was applied. ${ }^{23}$

\subsubsection{Blood sampling and biomarker analysis}

The participants provided nonfasting blood samples, obtained from the antecubital vein in BD vacutainer tubes with no additive (Becton, Dickinson and Company, Franklin Lakes, NJ). Serum was transferred to Supelco glass vials (Sigma-Aldrich Norway AS, Oslo, Norway) with Pasteur glass pipettes. The serum samples were stored at $270^{\circ} \mathrm{C}$ and thawed only once for biomarker analyses.

Serum levels of 92 inflammatory proteins were analysed by Protein Extension Array Technology (Proseek Multiplex Inflammation panel; Olink Bioscience, Uppsala, Sweden), which was used for relative quantification of proteins. Briefly, 3- $m \mathrm{~L}$ incubation mix containing 2 Proximity Extension Assay (PEA) probes, that is, antibodies equipped with single-strand DNA oligonucleotide, againsteach protein was mixed with 1 - $m L$ serum and incubated at $8^{\circ} \mathrm{C}$ overnight. The mixture was then added 96- $m L$ extension mix, containing PEA enzyme and PCR reagents, and incubated for 5 minutes at room temperature before the plate was transferred to a thermal cycler for 17 cycles of DNA amplification. A 96.96 Dynamic Array IFC (Fluidigm, South San Francisco, CA) was prepared and primed according to the manufacturer's instructions. Furthermore, $2.8 \mathrm{~mL}$ of sample mixture was added to $7.2-\mathrm{mL}$ detection mix in a new 96 -well plate, and $5 \mathrm{~mL}$ was loaded into the right side of the primed 96.96 Dynamic Array IFC. The unique primer pairs for each cytokine were loaded into the left side of the 96.96 Dynamic Array IFC, and the protein expression program was run in Fluidigm Biomark reader according to the instructions for Proseek.

Serum levels of 27 fatty acids (FAs) were analysed by Gas Chromatography with Flame lonization Detector (GC-FID) (Vitas AS, Oslo, Norway), which was used for absolute quantification of FAs. Serumsamples, thawedinfridgeovernight, arevortexed, centrifuged, and pipetted into vials. Internal standard (triheptadecanoin) is added, and samples are methylated with $3 \mathrm{~N} \mathrm{MeOH} \mathrm{HCl}$. Fatty acid methyl esters (FAMEs) are extracted with hexane, and then samples are neutralized with $3 \mathrm{~N} \mathrm{KOH}$ in water. After mixing and centrifuging, the hexane phase is injected into the GC-FID. Analysis is performed on a 7890A GC with a splitsplitless injector, a 7683B automatic liquid sampler, and flame ionization detection (Agilent Technologies, Palo Alto, CA). Separations are performed on a SP-2380 (30 $30.25-\mathrm{mm}$ internal diameter $30.25-\mathrm{mm}$ film thickness) column from Supelco.

\subsubsection{Pain sensitivity testing}

An experimental sensory testing session was conducted to assess responses to 3 stimulus modalities, applied in the following order: pressure, heat, and cold-pressor. The test protocol was based on methodology adapted from the sixth Troms $\varnothing$ Study, demonstrating feasibility. ${ }^{33,35}$

\subsubsection{Cold-pressor test}

Adolescents were asked to submerge their nondominant hand and wrist in a 13-L plexiglass container connected with a circulating cold $\left(3^{\circ} \mathrm{C}\right)$ water bath (Julabo PF40-HE; JULABO Labortechnik GmbH, Seelbach, Germany) for as long as they were able to, up to a maximum of 105 seconds (cold-pressor test). Time from immersion of hand and wrist to withdrawal was recorded as the cold-pressor pain tolerance (CPT).

\subsubsection{Heat pain threshold and tolerance}

Heat pain threshold and tolerance were assessed using a MEDOC ATS somatosensory stimulator with a $30330-\mathrm{mm}$ thermode (Medoc Ltd, Ramat Yishai, Israel). Heat stimuli were applied to the volar surface of the adolescent's nondominant forearm, starting at a baseline temperature of $32^{\circ} \mathrm{C}$, increasing by $1^{\circ} \mathrm{C} / \mathrm{s}$, to a maximum temperature of $50^{\circ} \mathrm{C}$. During pain threshold assessment, adolescents were instructed to press a button as soon as the sensation changed from warmth to pain, at which point the thermode returned to baseline at a rate of $8^{\circ} \mathrm{C} / \mathrm{s}$. A total of 3 trials were conducted, and the second and third trials were averaged for use in further analyses. To assess heat pain tolerance, the same stimulus procedure was used, but this time, the participants were instructed to press the button when they were unable to tolerate the pain any further. Two tolerance trials were conducted, and the average of these was used in further analysis.

\subsubsection{Pressure pain threshold and tolerance}

Threshold and tolerance assessments were made at 2 sites on the participants' nondominant arm: on the cuticle of the ring fingernail and on the trapezius muscle, midway between the shoulder joint and the neck. Pressure pain threshold and tolerance were assessed using a handheld computerized pressure algometer with a circular $1-\mathrm{cm}^{2}$ probe (AlgoMed, Medoc Ltd.). All stimuli started at $0 \mathrm{kPa}$ and increased by 30 $\mathrm{kPa}$ per second, up to a maximal pressure of $1000 \mathrm{kPa}$. For pressure pain threshold assessment, the participant was 
instructed to press a button when the sensation changed from pressure to pain, at which point the probe was removed. A total of 3 thresholds were obtained at each site, and the average of the second and third measurements was used in further analyses. Forpain toleranceassessments, the participants were instructed to press the button when they were unable to withstand the pain. Two tolerance assessments were made at each site (finger and trapezius). The average of these 2 measurements was used in further analyses.

\subsection{Outcomes}

The primary outcome was CPT chosen based on previous research showing the relationship between hs-CRP and widespreadhyperalgesiainapopulation-based cohort. ${ }^{1,30}$ Secondary outcomes included heat and pressure pain threshold and tolerance.

\subsection{Statistical analysis}

Statistical analysis was conducted using $R$ version 3.4.3 ( $R$ Foundation for Statistical Computing, Vienna, Austria). Statistical significance was set at the 0.05 level. To correct for multiple testing, a Benjamini-Hochberg false discovery rate (FDR) of 0.05 was applied. ${ }^{5}$ Biomarkers detected in $\$ 70 \%$ of the samples were included in the statistical analyses. Serum levels were $z$-score standardized using the population mean and SD. We modelled the effect of biomarkers on CPT with Cox proportional hazard regressions models with endurance time as the timescale and withdrawal of the hand from the water bath as the event and the maximum time ( 105 seconds) as the censoring point. Initially, we tested for sex interactions in models including the biomarker, sex, and sex 3 biomarker as predictors. For biomarkers where the sex interaction term was significant at $P, 0.05$ (uncorrected), all further analyses were stratified by sex. For biomarkers with nonsignificant sex interaction, the sex interaction term was omitted in further analyses. First, we modelled the crude risk for each biomarker, adjusting for sex in nonstratified analyses (model 1). Second, these analyses were repeated, adjusting for putative confounders (model 2 ):
BMI, tobacco use, alcohol consumption, depressive symptoms, and physical activity outside school. Third, in post hoc analyses, we modelled biomarker effects on secondary outcomes, heat and pressure pain threshold, and tolerance at fingernail and trapezius with linear regression and Cox proportional hazard regressions models, respectively. Only biomarkers that were significantly associated with CPT were included in these analyses. Finally, we investigated the discriminatory power of the proportional hazards model of the biomarker candidates significant in the primary analysis, which was assessed by the concordance probability (cindex) defined by Gönen and Heller ${ }^{15}$ using the GPE-package in $\mathrm{R}$. The $\mathrm{c}$-index is most familiar from logistic regression, where it is also known as the area under the receiveroperating curve. A c-index of 1 corresponds to a model with perfect discrimination and a value of 0.5 corresponds to a model with no discriminative ability.

\subsection{Ethics}

Data collection for The Troms $ø$ Study: Fit Futures was approved by the Regional Committee for Medical and Health Research Ethics (reference number 2011/1702/REK-Nord). The study procedures were conducted in accordance with the Declaration of Helsinki. Before any examination, written informed consent was obtained from the participants and, for those , 16 years, their guardian.

\section{Results}

\subsection{General description of sample}

Descriptive characteristics of the participants in the study are shown in Table 1. Half the participants (53\%) completed the coldpressor test without withdrawing their hand before the test ended. We observed sex differences where $53.2 \%$ girls vs $42.2 \%$ boys withdrew their hand before time was up ( $P 50.002)$. In the entire study population lower CPT was found among tobaccousers compared with nonusers $(P, 0.001)$, as well as among participants who were physically inactive outside school compared with physically active ( $P 50.001)$. Depressive symptoms and BMI were not associated with CPT.

Table 1

Descriptive characteristics of cold-pressor tolerance (CPT) groups. Fit Futures.

\begin{tabular}{|c|c|c|c|c|c|c|}
\hline & \multicolumn{2}{|c|}{ Girls (n 5 385) } & \multicolumn{2}{|c|}{ Boys (n 5 436) } & \multicolumn{2}{|c|}{ Both (n 5 821) } \\
\hline & $\begin{array}{l}\text { CPT <105 s } \\
\text { (n } 5 \text { 201) }\end{array}$ & $\begin{array}{l}\text { CPT } 5 \text { 105 } \\
\text { (n } 5 \text { 184) }\end{array}$ & $\begin{array}{l}\text { CPT <105 s } \\
\text { (n } 5 \text { 186) }\end{array}$ & $\begin{array}{l}\text { CPT } 5 \text { 105s } \\
\text { (n } 5250)\end{array}$ & $\begin{array}{l}\text { CPT <105 s } \\
\text { (n } 5 \text { 387) }\end{array}$ & $\begin{array}{l}\text { CPT } 5105 \mathrm{~s} \\
\text { (n } 5 \text { 434) }\end{array}$ \\
\hline Body mass index (BMI), kg/m², mean (SD) & $21.8(3.5)$ & $22.9(4.7)$ & $22.5(4.4)$ & $22.2(3.6)$ & $22.1(4.0)$ & $22.5(4.1)$ \\
\hline \multicolumn{7}{|l|}{ Tobacco use, $n(\%)$} \\
\hline Never users of any tobacco & $108(58.1)$ & $133(75.6)$ & $84(47.7)$ & $159(67.9)$ & $191(53.1)$ & $292(71.2)$ \\
\hline Ever users of snuff only & $40(21.5)$ & $29(16.5)$ & $46(26.4)$ & $42(18.0)$ & $86(23.9)$ & $71(17.3)$ \\
\hline $\begin{array}{l}\text { Ever smokers only } \\
\text { Ever users or potn snum ana smoke }\end{array}$ & $\begin{array}{l}14(7.5) \\
24(72 . y)\end{array}$ & $\begin{array}{l}5(2.8) \\
y(0.1)\end{array}$ & $\begin{array}{l}8(4.6) \\
3 /(21.3)\end{array}$ & $\begin{array}{l}5(2.1) \\
\angle \measuredangle(12 . U)\end{array}$ & $\begin{array}{l}22(6.1) \\
\text { bi (16.y) }\end{array}$ & $\begin{array}{l}10(2.5) \\
3 /(y .0)\end{array}$ \\
\hline Alcohol use (hazardous), $\mathrm{n}(\%)$ & $59(29.6)$ & $38(20.7)$ & $33(18.1)$ & $40(16.2)$ & $92(24.1)$ & $78(18.1)$ \\
\hline Emotional distress, n (\%), HSCL-10 \$1.85 & $29(14.6)$ & $26(14.1)$ & $19(10.3)$ & $11(4.5)$ & $48(12.5)$ & $37(8.6)$ \\
\hline \multicolumn{7}{|l|}{ Physical activity level in the past year, $n(\%)$} \\
\hline Sedentary & $26(13.1)$ & $21(11.4)$ & $74(40.2)$ & $55(22.3)$ & $100(26.1)$ & $76(17.7)$ \\
\hline Light physical activity & $90(45.2)$ & $62(33.7)$ & $46(25.0)$ & $54(21.8)$ & $136(35.5)$ & $116(26.9)$ \\
\hline Moderate physical activity & $59(29.6)$ & $59(32.1)$ & $41(22.3)$ & $60(24.3)$ & $100(26.1)$ & $119(27.6)$ \\
\hline Vigorous physical activity & $24(12.1)$ & $42(22.8)$ & $23(12.5)$ & 78 (31.6) & 47 (12.3) & $120(27.8)$ \\
\hline
\end{tabular}



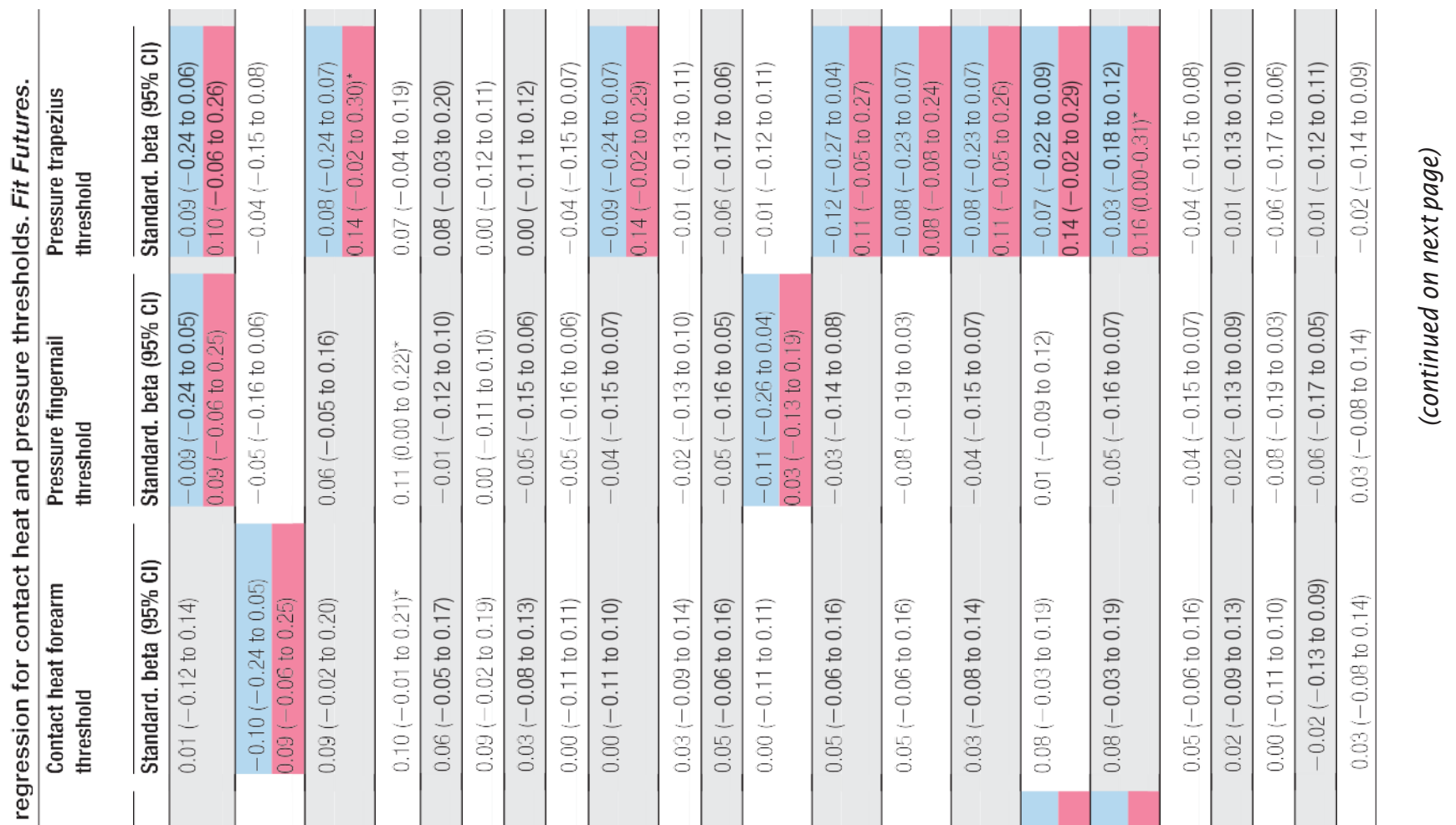

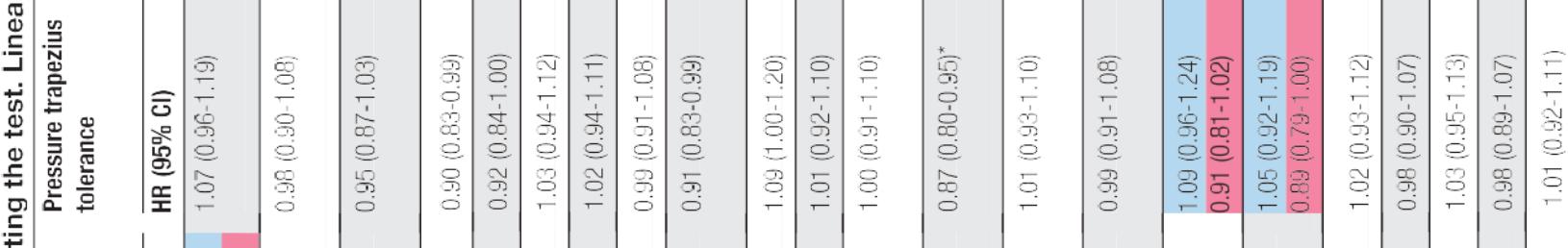

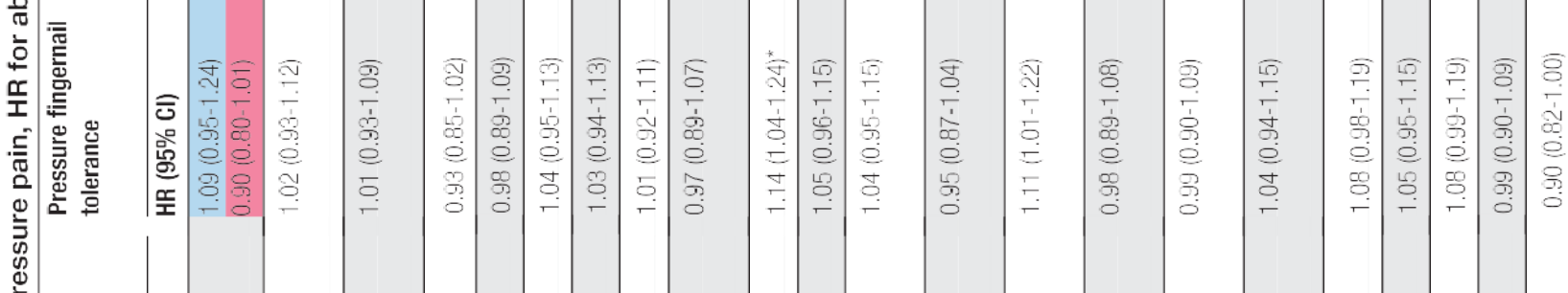
言

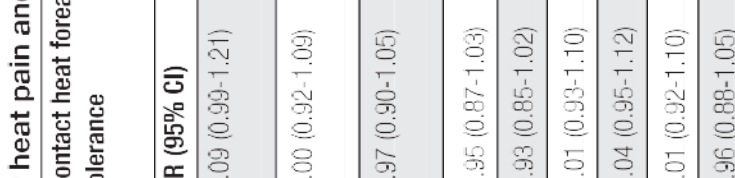
ㄴ 음

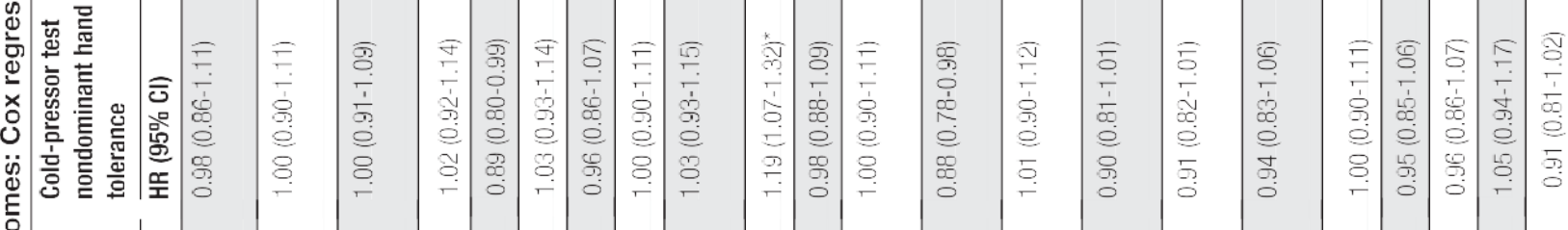
总

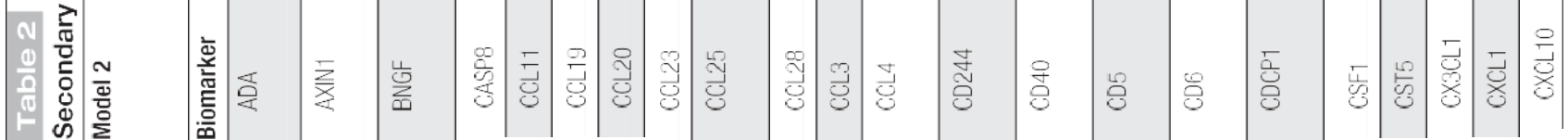




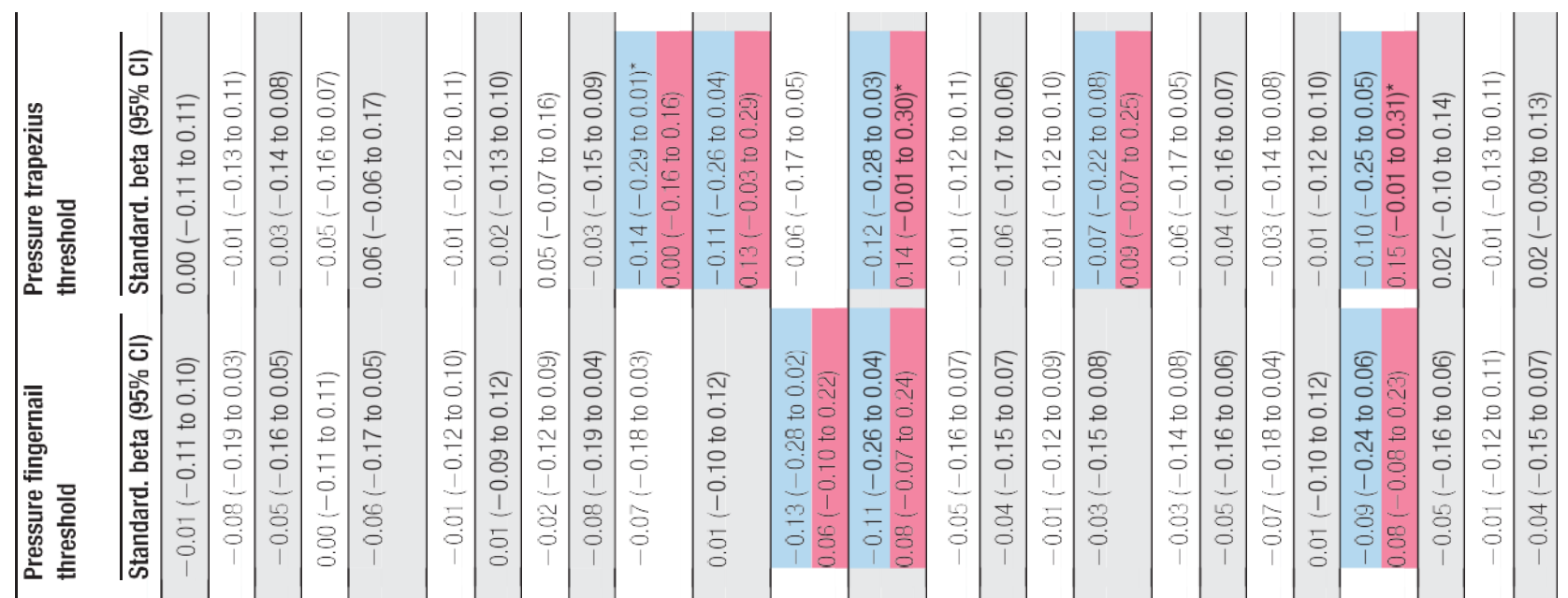

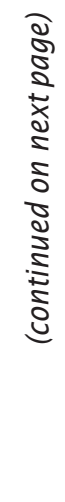

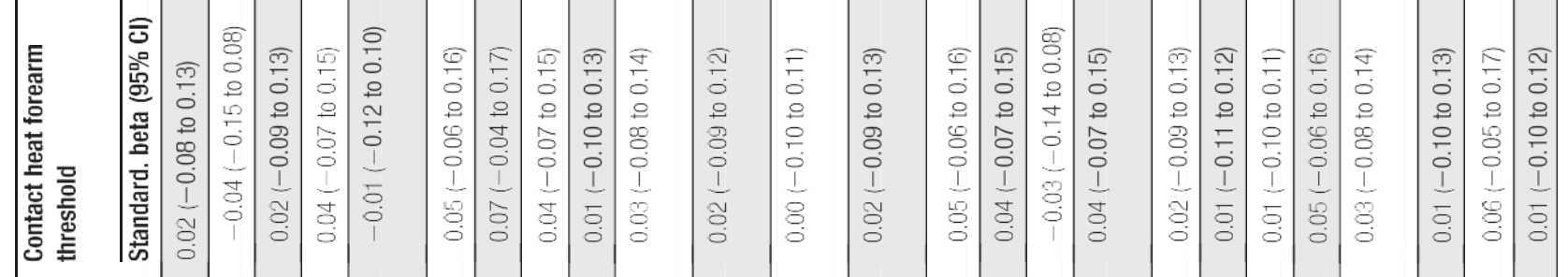

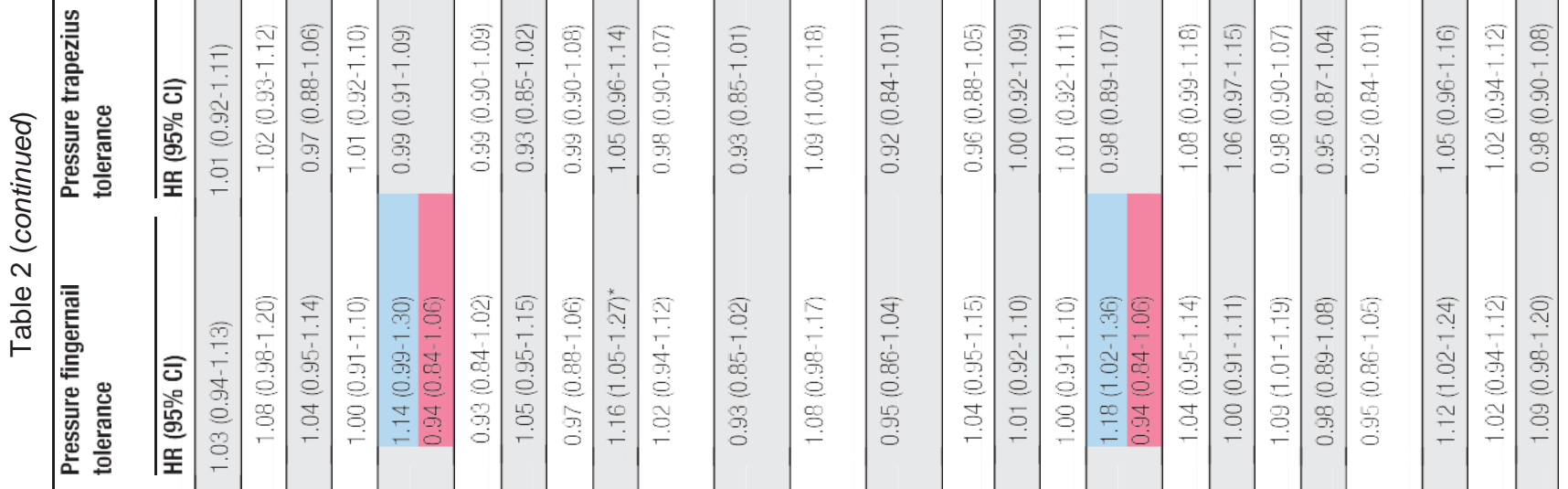
III InI

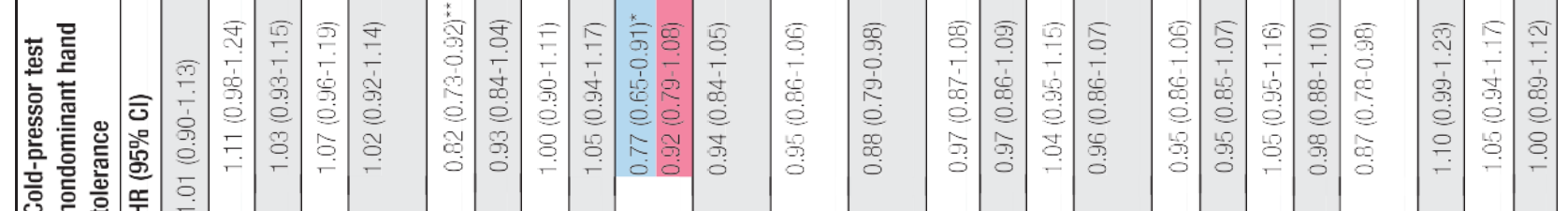

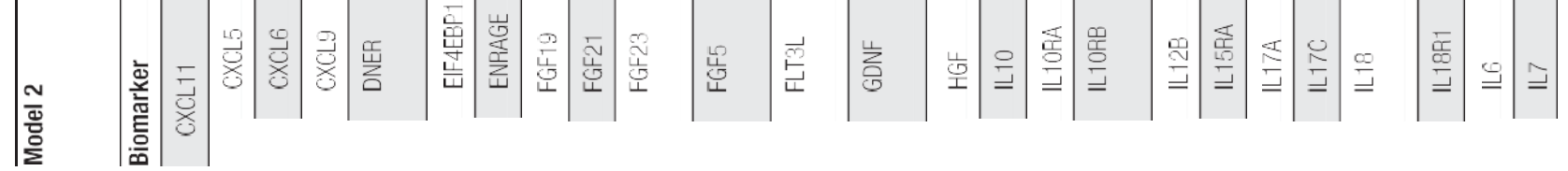




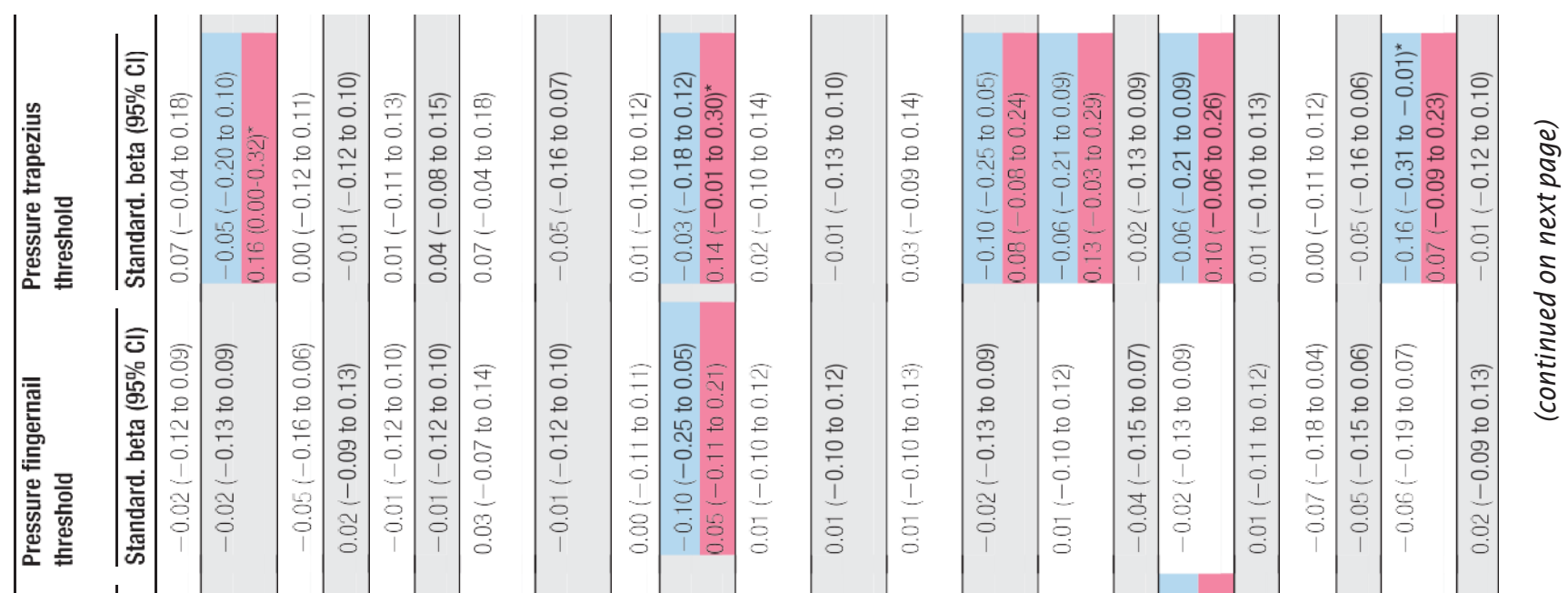

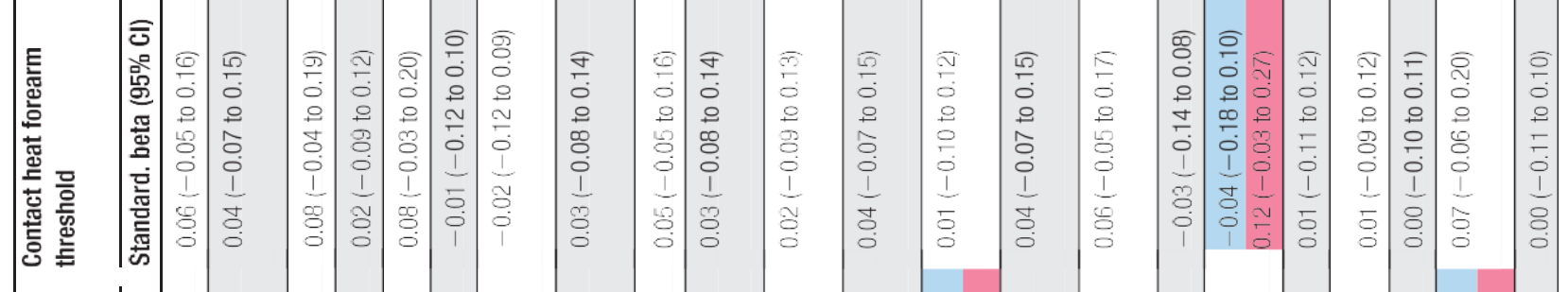

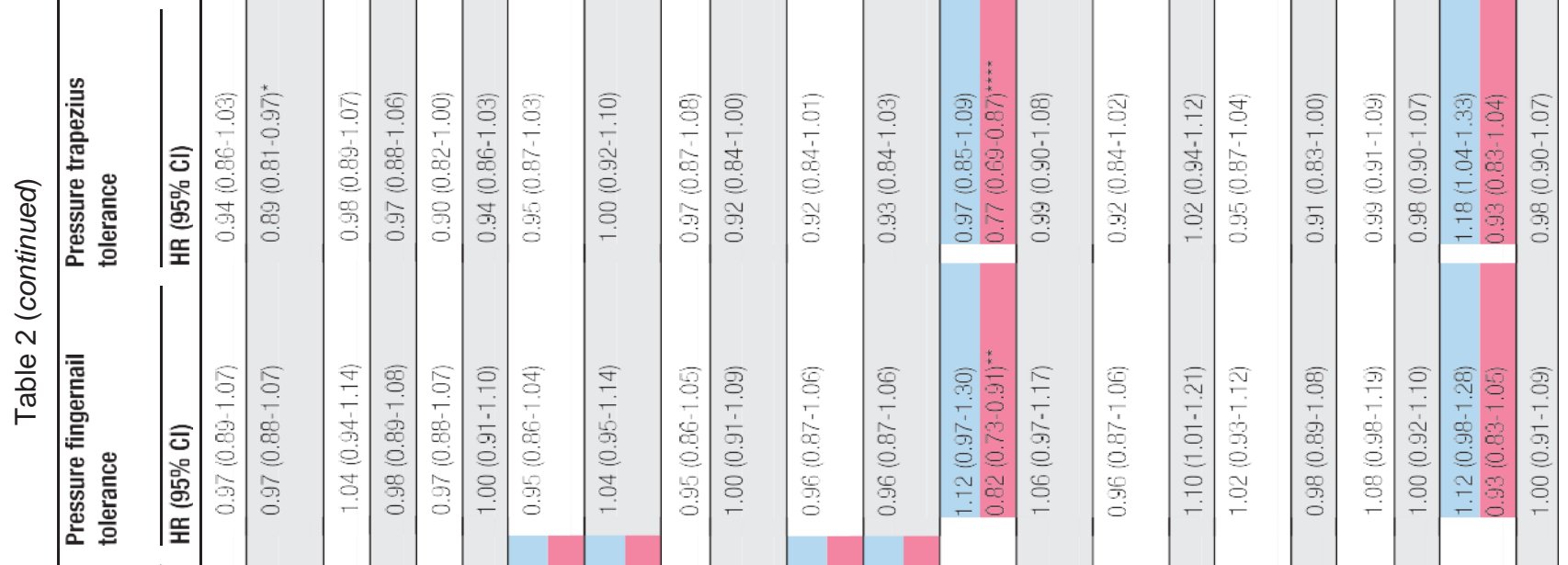

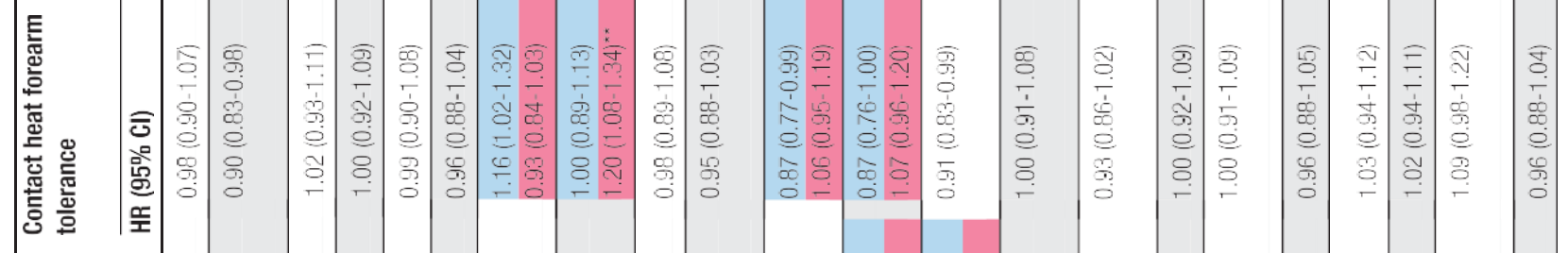

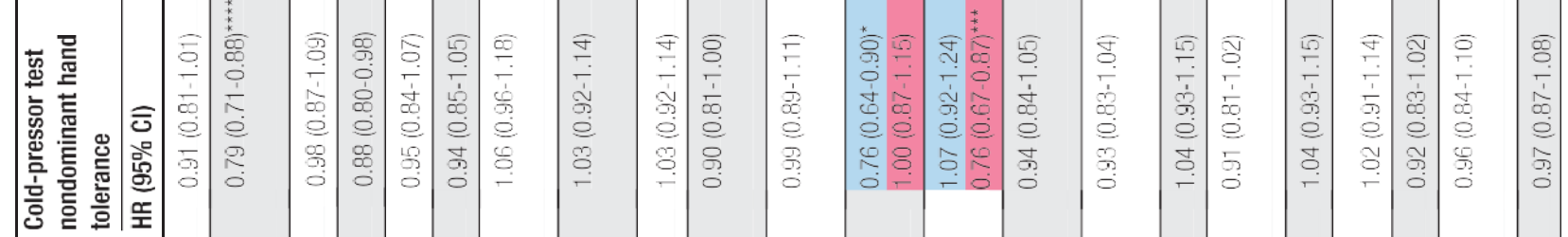

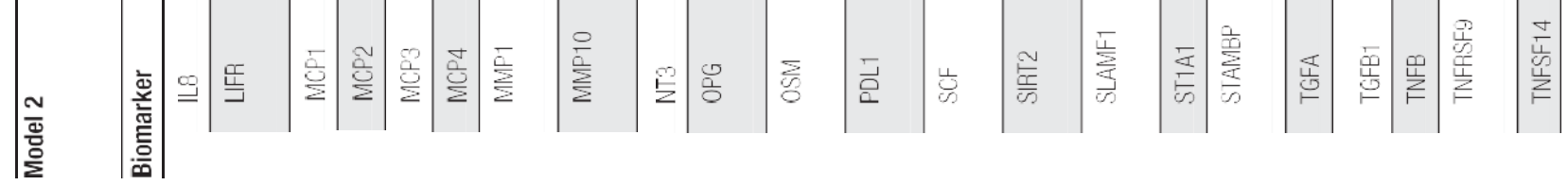




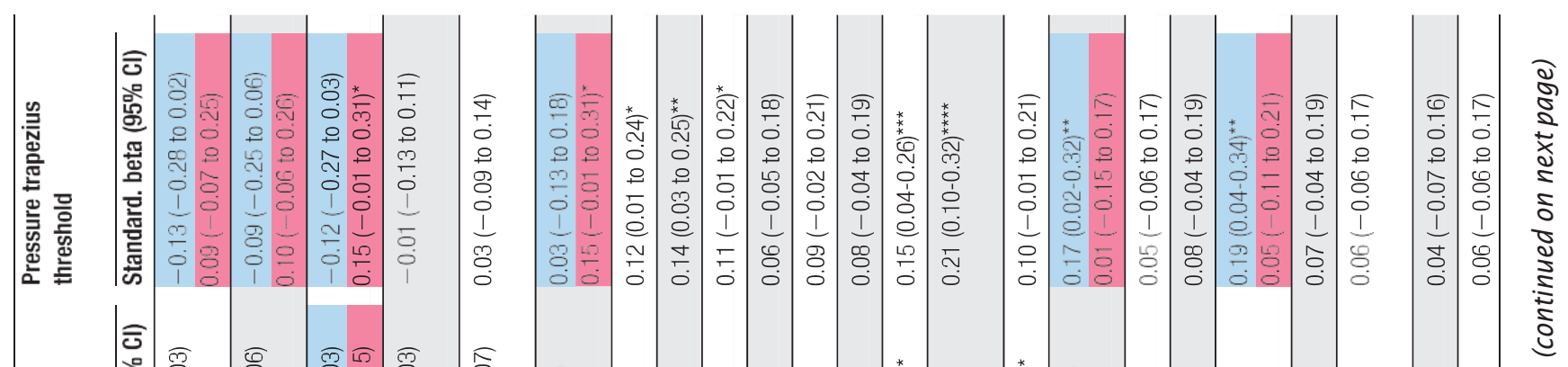

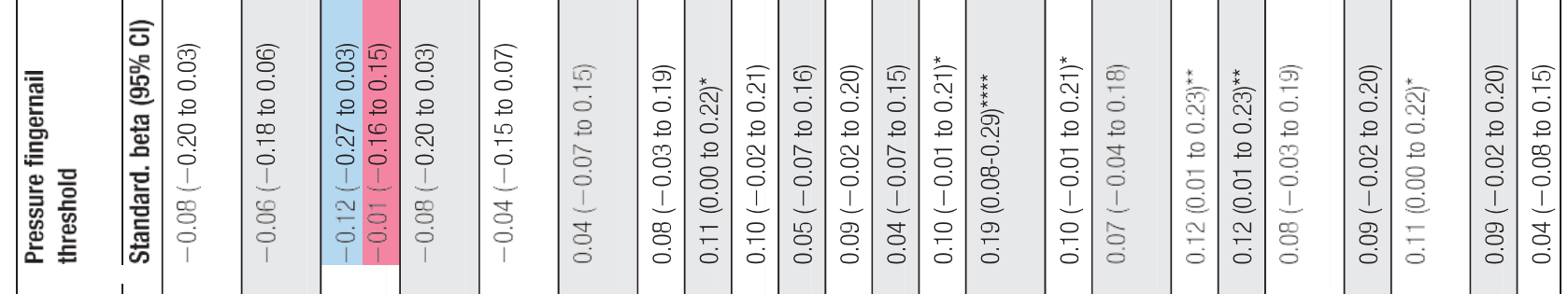

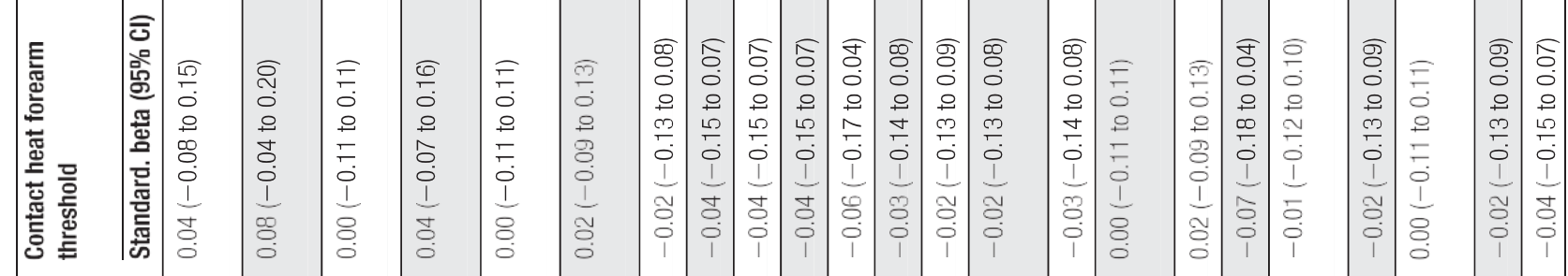

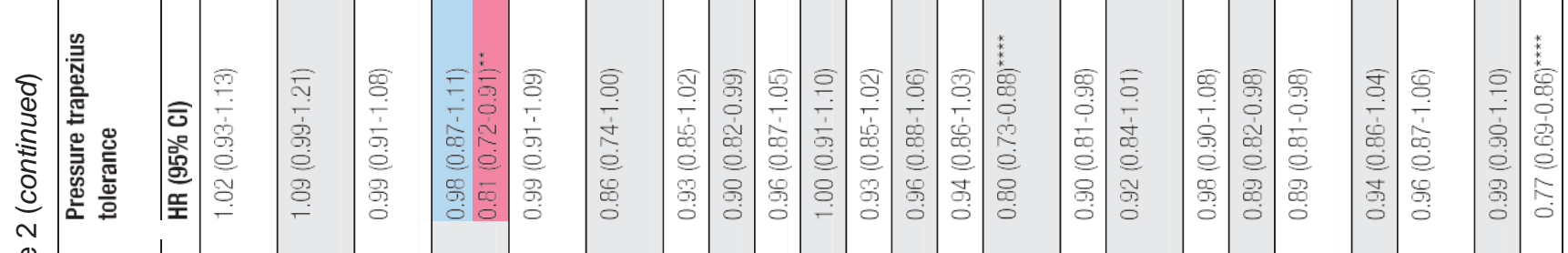
竞 II I I I I III

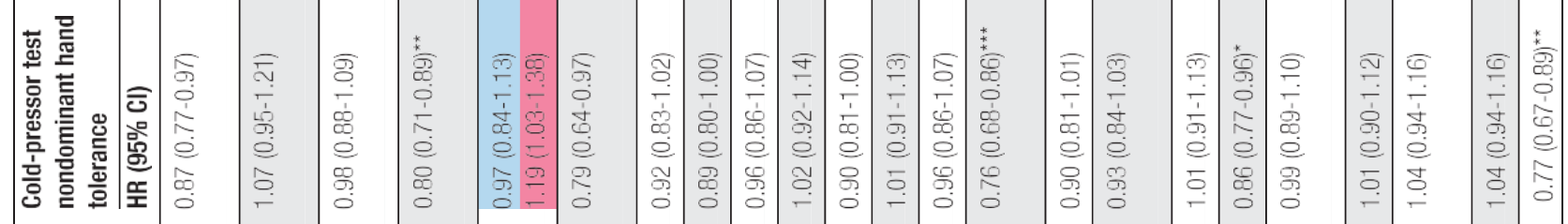

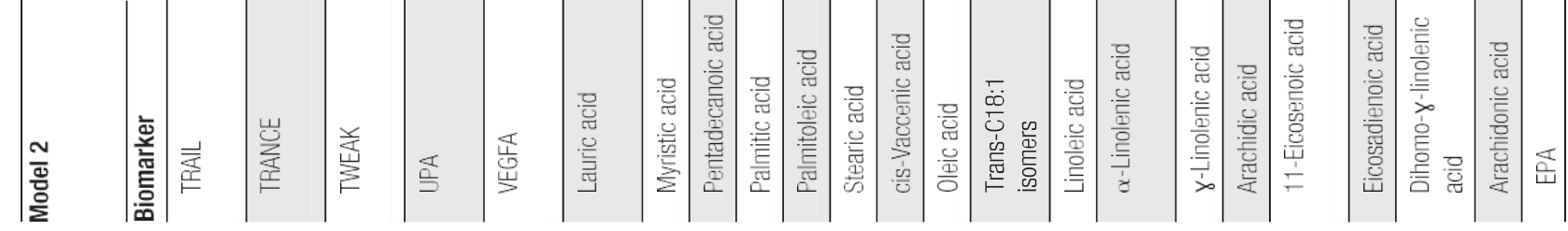


In total, 74/92 proteins and 27/27 FAs were detectable in $\$ 70 \%$ of the sample and were included in the analyses. Results of Cox regression analysis, unadjusted model revealed a relationship of 12 proteins and 6 FAs with CPT after correction for FDR (Fig. 2). Of these, all FAs and 10 proteins reduced the risk for aborting the cold-pressor test, ie, higher biomarkers levels were associated with increased CPT, whereas 2 biomarkers (CCL28 and FGF21) were associated with lower CPT. The concordance probability (cindex) of the 18 biomarkers predicting CPT was estimated to 0.65 (SE 5 0.012) indicating fair discriminatory power. The above relationships remained significant after adjusting for $\mathrm{BMI}$, tobacco or alcohol use, depression, and physical activity (Table 2).

\subsection{Biomarkers correlating with heat and pressure pain threshold and tolerance}

Biomarkers that were significantly associated with CPT were tested for associations with heat and pressure pain threshold and tolerance. Although the number of statistically significant associations was lower than in the regression model for the primary outcome, it is notable that the direction of effect was identical for all biomarkers when tested for heat and pressure pain tolerance (Figs. 2 and 3). Despite of more heterogeneous results for heat and pressure pain thresholds, all 6 FAs shown in Figure 4 were associated with higher thresholds for heat pain (although they did not reach significance). Results from the adjusted Cox regression model for heat and pressure pain threshold and tolerance are shown in Table 2. Correlations between biomarkers are shown in Figure 5.

\section{Discussion}

This large population-based study among adolescents revealed a relationship between 18 inflammation-related serum biomarkers and pain sensitivity. Overall, 12 proteins and 6 FAs were statistically significant associated with CPT. These relationships remained unchanged after adjusting for BMI, tobacco or alcohol use, depression, and physical activity. Furthermore, results were remarkably similar for 2 otherpain tolerance measures, strengthening the generalizability of our findings.

Pain is considered as 1 of 4 cardinal signs of inflammation, and mediators of the inflammatory response are well known to modulate both short- and long-term pain sensitivity. ${ }^{13}$ The close relationship between nociceptors and immune cells is evidenced by the shared expression of many cytokine-, growth factor- (GF), and toll-like receptors. ${ }^{28} \mathrm{~A}$ wide range of molecules can affect pain sensitivity, including ions, prostaglandins, hormones, GFs, cytokines, and lipids, ${ }^{16,28}$ thus complicating the search for correlations between single biomarkers and pain tolerance. Transmission from animals to human research is also challenging. In our approach, we simultaneously screened for 119 inflammation-related proteins and FAs and detected their association with pain sensitivity. Cytokines involved in the regulation of the innate immune system, cellular processes, and signal transduction represented our main findings. Cytokines are produced as a response to pathogens orduring injury and include both inflammatory and resolving mediators. This response not only recruit immune cell, but can also have a long-term effect on the sensitivity of nociceptors by interactions with surface receptors on the neuron. Interestingly, many of these cytokines have previously been associated with pain-eg, stem cell factor, ${ }^{26}$ programmed cell death ligand-1 (PD-L1), ${ }^{9} \mathrm{LL}-18,{ }^{43}$ 
- CPT - HPTO - PPTO_Finger - PPTO_Shoulder

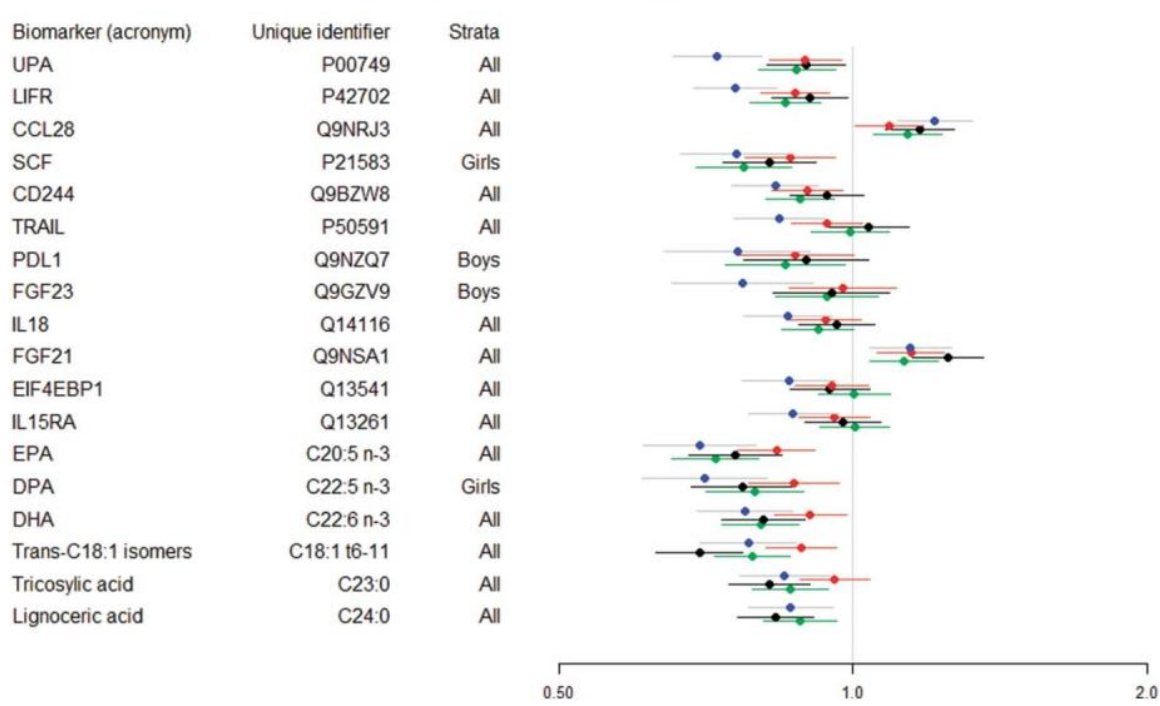

Figure 2. Forest plot showing the hazard ratio (HR) and 95\% Cl for cold-pressor pain tolerance (CPT), and heat and pressure pain tolerance, Fit Futures. Serum levels of the biomarkers were $z$-score standardized. *Unique identifier is either the protein number in accordance with the UniProt Knowledgebase or the carbon chain number of the fatty acid. Only variables significantly associated with CPT afterBenjamini-Hochberg false detection rate (FDR-adjusted) with threshold, $P \#$ 0.05 , are shown. $\mathrm{Cl}$, confidence interval.

and elF4EBP1. ${ }^{22}$ Adding to the complexity, peripheral and local concentrations can have opposite effects on pain sensitivity (ie, leukemia inhibitory factor receptor (LIFR)). ${ }^{25}$ Urokinase-type plasminogen activator showed the strongest positive association with CPT. Urokinase-type plasminogen activator has an important role in the regulation of inflammation, immunity, and coagulation through interactions with urokinase-type plasminogen activator receptor. Urokinase-type plasminogen activator/ urokinase-type plasminogen activator receptor promotes tissue remodeling through activation of plasmin and metalloproteinases and recruits circulating leukocytes through interactions with endothelial integrins. ${ }^{7}$ Stem cell factor and PD-L1, secreted by endothelial cells and dorsal root ganglions, respectively, disturb pain signal transduction in the sensory neuron by affecting ion channels. Stem cell factor interacts with the receptor c-Kit, expressed on neurons. The hyperalgestic effect of this interaction depends on the TRPV1 cation channel. ${ }^{26}$ PD-L1, typically secreted by cancer cells, activates the receptor PD-1 and inhibits neuronal activities by modulating sodium and potassium channels. ${ }^{9}$ The protein with the strongest negative association with CPT in our study was C-C motif chemokine 28 (CCL28). CCL28 is expressed by mucosa-associated epithelial cells in the gut, trachea, and salivary glands. It plays dual roles in mucosal immunity as an antimicrobial agent and as a chemoattractant for CC-chemokine receptor 10 (CCR10)-expressing immune cells. The expression is modulated by proinflammatory cytokines and bacterial products such as lipopolysaccharides. ${ }^{18}$ Thus, epithelial inflammation is associated with CCL28 production.

The acute inflammatory response is counterbalanced by a complex process termed resolution where apoptosis of neutrophils and their subsequent clearance herald potent

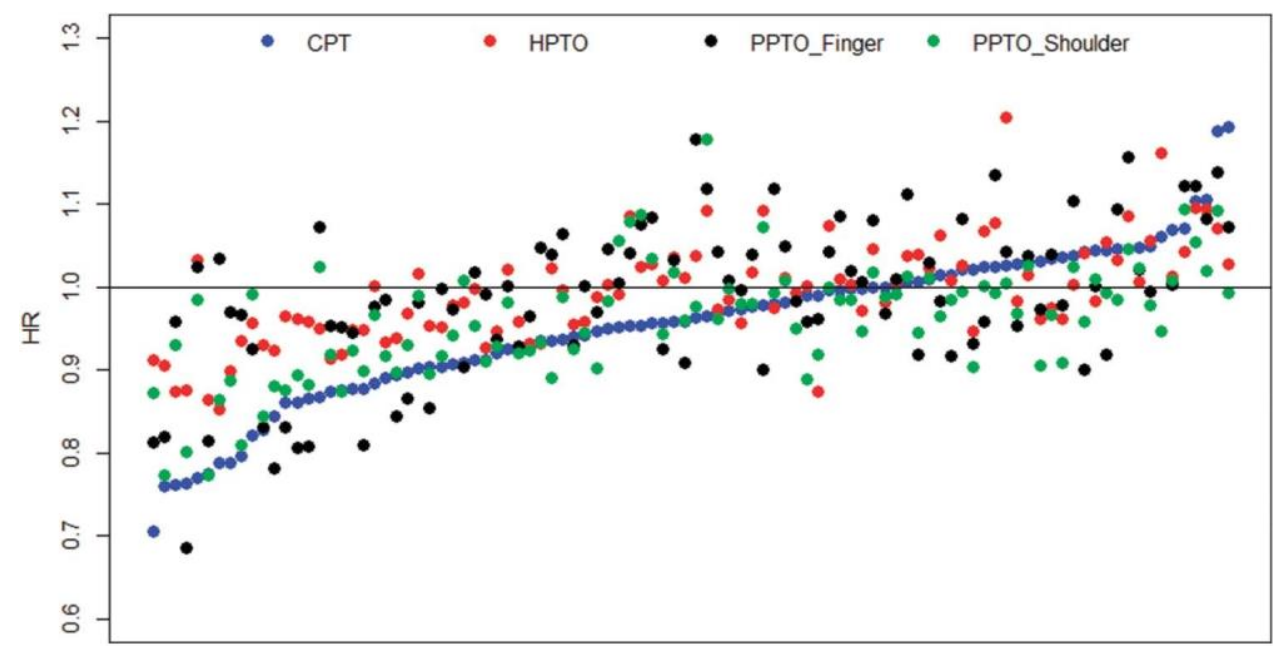

Figure 3. Hazard ratios (HRs) for aborting the test for cold-pressor pain tolerance (CPT), and heat and pressure pain tolerance sorted by HR of CPT, Fit Futures. Serum levels of the biomarkers were $z$-score standardized. All investigated biomarkers are shown. 


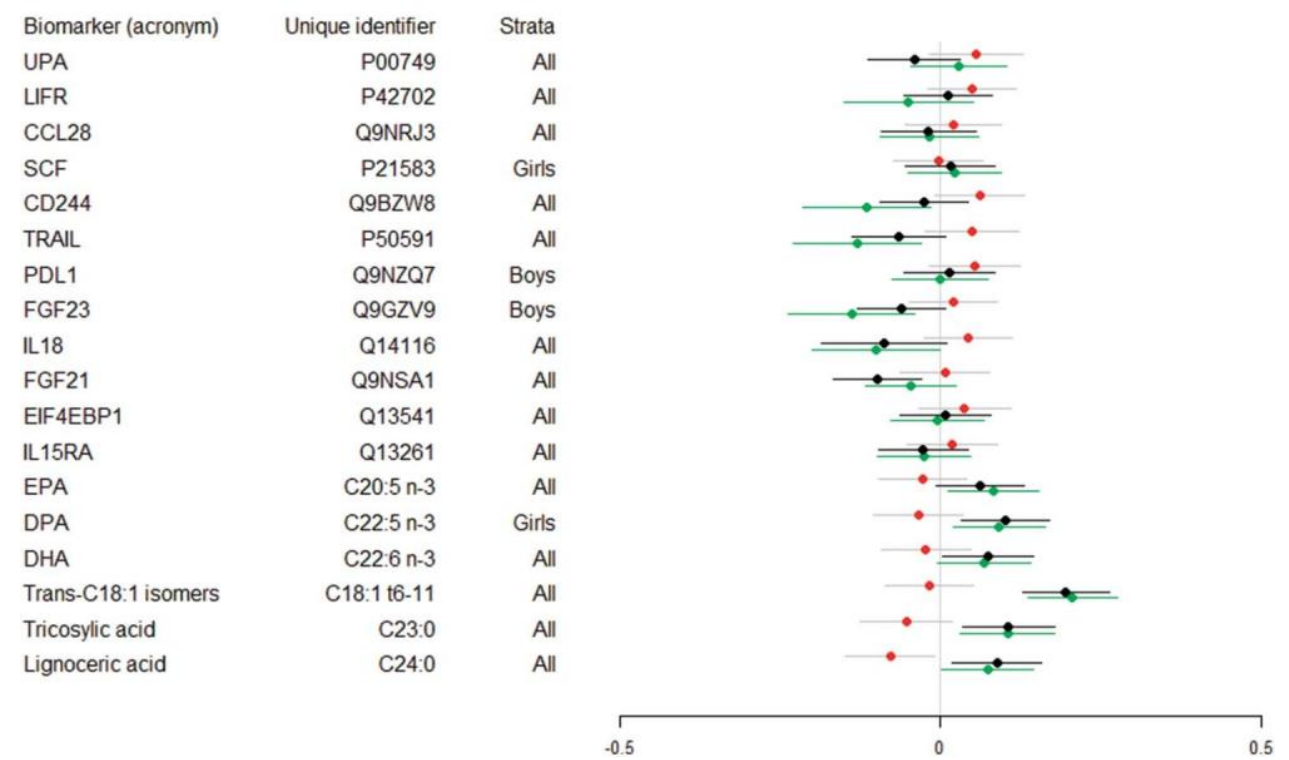

Figure 4. Forest plot showing standardized betas and $95 \% \mathrm{Cl}$ for heat and pressure pain threshold, Fit Futures. Serum levels of the biomarkers were $z$-score standardized. *Unique identifier is either the protein number in accordance with the UniProt Knowledgebase or the carbon chain number of the fatty acid. Only variables significantly associated with CPT after Benjamini-Hochberg false detection rate (FDR adjusted) with threshold, $P \# 0.05$, are shown. $\mathrm{Cl}$, confidence interval; CPT, cold-pressor pain tolerance.

anti-inflammatory mechanisms. Endogenous proteins/peptides and lipid mediators including $v$-3 FA derived "specialized proresolution mediators" such as lipoxins, resolvins (Rvs), maresins, and protectins released by a number of cell types orchestrate the resolution of inflammation and tissue repair. Resolvins dampens pain sensation through inhibition of the

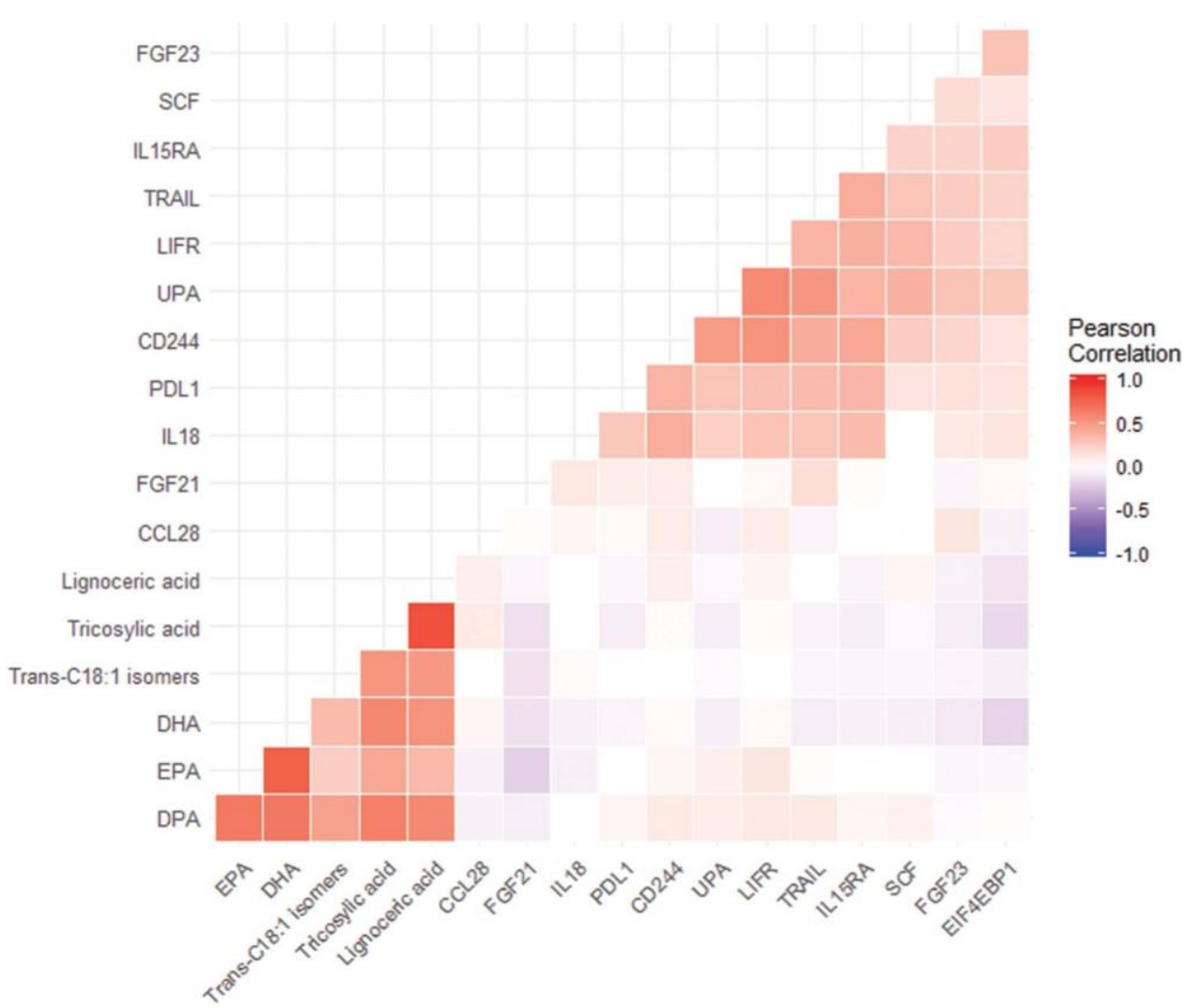

Figure 5. Correlations among biomarkers. Color scale:red 5 highest positivecorrelation, white 5 nocorrelation, blue 5 highest negative correlation. Serum levels of the biomarkers were z-scorestandardized. Onlyvariablessignificantlyassociated withCPT afterBenjamini-Hochbergfalse detection rate (FDRadjusted) with threshold, $P \# 0.05$, are shown. CPT, cold-pressor pain tolerance. 
release of inflammatory mediators from immune cells, inhibition of ion channels, and by affecting signaling cascades in dorsal root ganglion and presynaptic and postsynaptic neurons. The $v-3$ FAs docosahexaenoic acid (DHA) and eicosapentaenoic acid (EPA) originate the D- and E-series Rvs, respectively. Recent studies have highlighted the importance of RvD1 and RvE1 in acute and persistent tissue inflammation in animal models. ${ }^{6,41}$ The RvD1-6, neuroprotection D1, actively reverses inflammatory pain in mice. ${ }^{4}$ We found strong positive associations of DHA and EPA with higher CPT suggesting a protective role of these FAs in the modulation of pain sensitivity. Clinical trials have previously shown that $v$-3 FAs supplementation is protective in selected phenotypes (eg, rheumatoid arthritis). ${ }^{12}$ Our findings suggest that RCTs investigating the effect of dietary supplements on chronic pain and hyperalgesia are warranted.

In addition, this study showed lower pain tolerance among girls, smokers, and/or snuff users, as well as among participants physically inactive outside school. Sex differences are commonly reported in pain research, providing evidence of higher prevalence of widespread pain as well as greater sensitivity to experimental pain among girls/women. ${ }^{14}$ We also found some sex interactions in the association between biomarkers and pain sensitivity (stem cell factor, PD-L1, and FGF23). Interestingly, the function of these proteins have all been demonstrated to be regulated downstream ofestrogen signaling cascades. ${ }^{8,14,42}$ However, the relationship between hormones, inflammatory biomarkers, and pain sensitivity needs further studies.

\subsection{Study strengths and limitations}

The strengths of our study are its size and the population-based design including high attendance rates in both sexes. Because of participants' age and exclusion of adolescents with inflammatory disease or ongoing infection, these data are considered valid for the investigation of inflammation-related pain mechanisms. However, our study has some limitations. Because of the cross-sectional design, conclusions about causality cannot be drawn. Second, no adjustment was made for unmeasured factors, such as dietary status possibly related to socioeconomic status of parents (confounding). Nevertheless, - $50 \%$ of the variance in cold-pressor responses can be explained by genetic factors ${ }^{27}$; therefore, the relative contribution of the environment is limited. Third, nonfasting blood samples may be subject to bias. Fourth, our study was unable to demonstrate a pivotal role of IL-6, IL- $1 b$, and TNF- $a$ in pain sensitivity despite preclinical evidence that these cytokines sensitize the peripheral nerve endings that lead to hyperalgesia. Fifth, our study was limited to a multiplex panel of preselected inflammatory biomarkers, and many putative pain-related biomarkers were not investigated.

\section{Conclusions}

In summary, we found a relationship between inflammationrelated biomarkers and pain tolerance in this cohort of young healthy individuals. Biomarkers with anti-inflammatory and analgesic effects predominated, suggesting that the development of prophylactic dietary or pharmaceutical treatments may be possible.

\section{Acknowledgments}

The authors thank the participants, the staff at the Clinical Research Unit at University Hospital of North Norway (UNN HF), and the Fit Futures administration for conducting the study. Thisstudy wasfunded by financial supportfrom theSouthEastern Norway Regional Health Authority. Emmanuel Bäckryd was supported by a grant from theNEURO Sweden.

\section{References}

[1] Afari N, Mostoufi S, Noonan C, Poeschla B, Succop A, Chopko L, Strachan E. C-reactive protein and pain sensitivity: findings from female twins. Ann Behav Med 2011;42:277-83.

[2] Atienza M, Ziontz J, Cantero JL. Low-grade inflammation in the relationship between sleep disruption, dysfunctional adiposity, and cognitive decline in aging. Sleep Med Rev 2018;42:171-83.

[3] Backryd E, Tanum L, Lind AL, Larsson A, Gordh T. Evidence of both systemic inflammation and neuroinflammation in fibromyalgia patients, as assessed by a multiplex protein panel applied to the cerebrospinal fluid and to plasma. J Pain Res 2017;10:515-25.

[4] Bang S, Xie YK, Zhang ZJ, Wang Z, Xu ZZ, Ji RR. GPR37 regulates macrophage phagocytosis and resolution of inflammatory pain. J Clin Invest 2018;128:3568-82.

[5] Benjamini Y, Hochberg Y. Controlling the false discovery rate: a practical and powerful approach to multiple testing. JRStat Soc Ser B 1995;57:11.

[6] Bento AF, Claudino RF, Dutra RC, Marcon R, Calixto JB. Omega-3 fatty acid-derived mediators 17(R)-hydroxy docosahexaenoic acid, aspirintriggeredresolvin D1 andresolvin D2 preventexperimental colitis in mice. J Immunol 2011;187:1957-69.

[7] Blasi F, Carmeliet P. uPAR: a versatile signalling orchestrator. Nat Rev Mol Cell Biol 2002;3:932-43.

[8] Carrillo-Lo' pez N, Roma' n-Garc'ıa P, Rodrí Iguez-Rebollar A, Ferna' ndezMart' inJL, Naves-D'iazM, Cannata-And'ıaJB. Indirect regulation of PTH by estrogens may require FGF23. J Am Soc Nephrol 2009;20:2009-17.

[9] Chen G, Kim YH, Li H, Luo H, Liu DL, Zhang ZJ, Lay M, Chang W, Zhang YQ, Ji RR. PD-L1 inhibits acute and chronic pain by suppressing nociceptive neuron activity via PD-1. Nat Neurosci 2017;20:917-26.

[10] Choghakhori R, Abbasnezhad A, Hasanvand A, Amani R. Inflammatory cytokines and oxidative stress biomarkers in irritable bowel syndrome: association with digestivesymptoms andquality of life. Cytokine2017;93: 34-43.

[11] Demartini KS, Carey KB. Optimizing the use of the AUDIT for alcohol screening in college students. Psychol Assess 2012;24:954-63.

[12] Di Giuseppe D, Wallin A, Bottai M, Askling J, Wolk A. Long-term intake of dietary long-chain n-3 polyunsaturated fatty acids and risk of rheumatoid arthritis: a prospective cohort study of women. Ann Rheum Dis 2014;73: 1949-53.

[13] Dubin AE, Patapoutian A. Nociceptors: the sensors of the pain pathway. J Clin Invest 2010;120:3760-72.

[14] FigueiraMI, CorreiaS, VazCV, Cardoso HJ, Gomes IM, Marques R, Maia CJ, SocorroS. Estrogens down-regulate the stem cell factor (SCF)/c-KIT system in prostate cells: evidence of antiproliferative and proapoptotic effects. Biochem Pharmacol 2016;99:73-87.

[15] Gönen M, Heller G. Concordance probability and discriminatory power in proportional hazards regression. Biometrica2005;92:965-70.

[16] Gangadharan V, KunerR.Painhypersensitivity mechanisms ataglance. Dis Model Mech 2013;6:889-95.

[17] Gerdle B, Ghafouri B, Ghafouri N, Backryd E, Gordh T. Signs of ongoing inflammation in female patients with chronic widespread pain: a multivariate, explorative, cross-sectional study of blood samples. Medicine (Baltimore) 2017;96:e6130.

[18] Hieshima K, Ohtani H, Shibano M, Izawa D, Nakayama T, Kawasaki Y, Shiba F, Shiota M, Katou F, Saito T, Yoshie O. CCL28 has dual roles in mucosal immunity as a chemokine with broad-spectrum antimicrobial activity. J Immunol 2003;170:1452-61.

[19] Hu XX, Wu YJ, Zhang J, Wei W. T-cells interact with B cells, dendritic cells, and fibroblast-like synoviocytes as hub-like key cells in rheumatoid arthritis. Int Immunopharmacol 2019;70:428-34.

\section{Conflict of interest statement}

The authors have no conflicts of interest to declare. 
[20] Ji RR, Berta T, Nedergaard M. Glia and pain: is chronic pain a gliopathy? PAIN 2013;154(suppl 1):S10-28.

[21] JiRR, Nackley A, HuhY, Terrando N, MaixnerW. Neuroinflammationand central sensitization in chronic and widespread pain. Anesthesiology 2018;129:343-66.

[22] Khoutorsky A, Bonin RP, Sorge RE, Gkogkas CG, Pawlowski SA, Jafarnejad SM, Pitcher MH, Alain T, Perez-Sanchez J, Salter EW, Martin L, Ribeiro-da-Silva A, De Koninck Y, Cervero F, Mogil JS, Sonenberg N. Translational control of nociception via 4E-binding protein 1. eLife 2015; 4:e12002.

[23] Larsson B, Ingul J, Jozefiak T, Leikanger E, Sund AM. Prevalence, stability, 1 -year incidence and predictors of depressive symptoms among Norwegian adolescents in the general population as measured by the Short Mood and Feelings Questionnaire. Nord J Psychiatry 2016;70:290-6.

[24] Linnman C, Appel L, Fredrikson M, Gordh T, Soderlund A, Langstrom B, Engler H. Elevated [11C]-D-deprenyl uptake in chronic Whiplash Associated Disorder suggests persistent musculoskeletal inflammation. PLoS One 2011;6:e19182.

[25] Metcalf $D$. Theunsolvedenigmas ofleukemiainhibitoryfactor. Stem Cells 2003;21:5-14.

[26] Milenkovic N, Frahm C, Gassmann M, Griffel C, Erdmann B, Birchmeier C, Lewin GR, Garratt AN. Nociceptive tuning by stem cell factor/c-Kit signaling. Neuron 2007;56:893-906.

[27] Nielsen CS, Knudsen GP, Steingrimsdottir OA. Twin studies of pain. Clin Genet 2012;82:331-40.

[28] Pinho-Ribeiro FA, Verri WA Jr, Chiu IM. Nociceptor sensory neuronimmune interactions in pain and inflammation. Trends Immunol 2017;38: 5-19.

[29] Ruiz-Nunez B, Pruimboom L, Dijck-Brouwer DA, Muskiet FA. Lifestyle and nutritional imbalances associated with Western diseases: causes and consequences of chronic systemic low-grade inflammation in an evolutionary context. J Nutr Biochem 2013;24:1183-201.

[30] Schistad El, Stubhaug A, Furberg AS, Engdahl BL, Nielsen CS. Creactive protein and cold-pressor tolerance in the general population: the Tromso Study. PAIN 2017;158:1280-8.

[31] Sorge RE, LaCroix-Fralish ML, TuttleAH, Sotocinal SG, Austin JS, Ritchie J Chanda ML, Graham AC, Topham L, Beggs S, Salter MW, Mogil JS. Spinal cord toll-like receptor 4 mediates inflammatory and neuropathic hypersensitivity in male but not female mice. J Neurosci 2011;31:15450-4.

[32] Sorge RE, Mapplebeck JC, Rosen S, Beggs S, Taves S, Alexander JK, Martin LJ, Austin JS, Sotocinal SG, Chen D, Yang M, Shi XQ, Huang H, Pillon NJ, Bilan PJ, Tu Y, Klip A, Ji RR, Zhang J, Salter MW, Mogil JS.
Different immune cells mediate mechanical pain hypersensitivity in male and female mice. Nat Neurosci 2015;18:1081-3.

[33] Stabell N, Stubhaug A, Flaegstad T, Nielsen CS. Increased pain sensitivity among adults reporting irritable bowel syndrome symptoms in a large population-based study. PAIN 2013;154:385-92.

[34] Sterling M, Elliott JM, Cabot PJ. The course of serum inflammatory biomarkersfollowing whiplash injury and their relationship to sensory and muscle measures: a longitudinal cohort study. PLoS One 2013;8: e77903.

[35] Tham SW, Palermo TM, Holley AL, Zhou C, Stubhaug A, Furberg AS, Nielsen CS. A population-based study of quantitative sensory testing in adolescents with and without chronic pain. PAIN 2016;157:2807-15.

[36] UceylerN, HauserW, Sommer C. Systematic review with meta-analysis: cytokines in fibromyalgia syndrome. BMC Musculoskelet Disord 2011; 12:245.

[37] Valdes AM, RavipatiS, Menni C, AbhishekA, Metrustry S, Harris J, Nessa A, Williams FMK, Spector TD, Doherty M, Chapman V, Barrett DA. Association of the resolvin precursor 17-HDHA, but not D- or E- series resolvins, with heat pain sensitivity and osteoarthritis pain in humans. Sci Rep 2017;7:10748.

[38] Vehof J, Zavos HM, Lachance G, Hammond CJ, Williams FM. Shared genetic factors underlie chronic pain syndromes. PAIN 2014;155: 1562-8.

[39] Williams FM, Spector TD, MacGregor AJ. Pain reporting at differentbody sites is explained by a single underlying genetic factor. Rheumatology (Oxford) 2010;49:1753-5.

[40] Winther A, Dennison E, Ahmed LA, Furberg AS, Grimnes G, Jorde R Gjesdal CG, Emaus N. The Tromso Study: Fit Futures: a study of Norwegian adolescents' lifestyle and bone health. Arch Osteoporos 2014;9:185

[41] Xu ZZ, Zhang L, Liu T, Park JY, Berta T, Yang R, Serhan CN, Ji RR. Resolvins RvE1 and RvD1 attenuate inflammatory pain via central and peripheral actions. Nat Med 2010;16:592-7; 591p following 597.

[42] Yang L, Huang F, Mei J, Wang X, Zhang Q, Wang H, Xi M, You Z. Posttranscriptional control of PD-L1 expression by 17 beta-estradiol via $\mathrm{PI} 3 \mathrm{~K} /$ Akt signaling pathway in ERalpha-positive cancer cell lines. Int $\mathrm{J}$ Gynecol Cancer 2017;27:196-205.

[43] Yoshida S, Hagiwara Y, Tsuchiya M, Shinoda M, Koide M, Hatakeyama H, Chaweewannakorn C, Yano T, Sogi Y, Itaya N, Sekiguchi T, Yabe Y, SasakiK, Kanzaki M, Itoi E. Involvement of neutrophils and interleukin-18 in nociception in a mouse model of muscle pain. Mol Pain 2018;14: 1744806918757286 